\title{
Effect of Fast Motion on Range Images Acquired by Lidar Scanners for Automotive Applications
}

\author{
Lutz Gröll and Andreas Kapp, Student Member, IEEE
}

\begin{abstract}
The scanning process of lidar scanners is extended over time. Hence, relative motion between sensor and object during the scanning leads to a motion-dependent blur, here called motion-scan effect. Due to this effect the lidar's distance map is deteriorated, which has a negative impact on the subsequent signal processing. This paper quantifies this effect and compares it to statistical errors. In order to compensate for the negative impact, motion models for a simple car model are formulated, and the model parameters are checked for identifiability. Identification of the parameters allows to compensate for the effect. Thus the accuracy and the control mode can be improved, which is particularly important for safety-critical applications, e.g., autonomous vehicles.
\end{abstract}

Index Terms-Compensation, identifiability, lidar scanner, optimal estimation, parameter estimation.

\section{INTRODUCTION}

$\mathbf{L}$ IGHT detection and ranging (lidar) sensors acquire a distance map of their field of view using the time-of-flight principle. The laser ray is deflected by a rotating mirror, and the sensor scans the complete field of view during one scan period. In contrast to this, range imagers instantaneously acquire a distance map.

Lidar scanners are used for the following:

a) as qualitative detectors to monitor work-places;

b) to quickly acquire a distance map with moderate resolution;

c) for slow, high-resolution surveying of buildings.

Application area b) comprises the following:

- dynamic mapping of streets [1];

- simultaneous localization and mapping [2];

- measuring of buildings [3];

- navigation of mobile robots under water [4], ashore [5], and airborne [6];

- autonomous vehicles [7];

- driver assistance systems, e.g., for collision avoidance, sometimes in a fusion approach with vision [8].

The two latter items are the focus of this work.

A good part of the application areas listed above can be abstracted as scanning a structured environment consisting of line segments, which moves relatively to the lidar sensor during scanning. For instance this is the case when scanning a moving car.

Manuscript received June 6, 2006; revised October 2, 2006. The associate editor coordinating the review of this manuscript and approving it for publication was Dr. Tulay Adali.

L. Gröll is with the Institut für Angewandte Informatik, Forschungszentrum Karlsruhe, 76021 Karlsruhe, Germany (e-mail: groell@iai.fzk.de).

A. Kapp is with the Institut für Mess-und Regelungstechnik, Universität Karlsruhe (TH), 76128 Karlsruhe, Germany (e-mail: kapp@mrt.uka.de).

Digital Object Identifier 10.1109/TSP.2007.893945

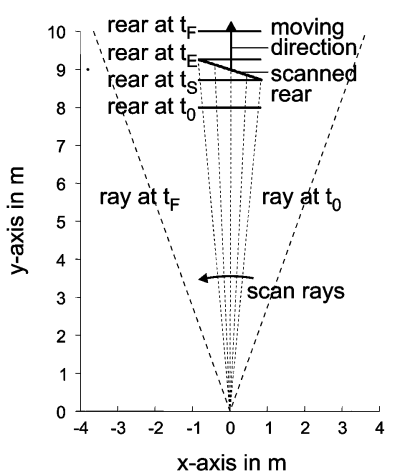

(a)

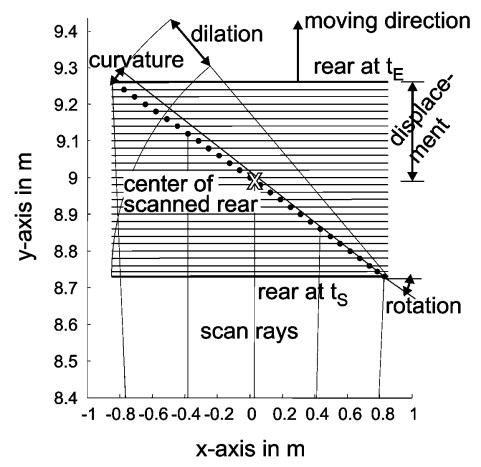

(b)
Fig. 1. Illustration of the motion-scan effect in (a) general view and (b) zoomed. A fast-moving line segment representing the rear of a car is scanned by the sensor located in the origin. The scanning indicated by scan rays starts at time $t_{0}$ and ends at $t_{F}$. At time $t_{S}$ the car is hit for the first time, at $t_{E}$ for the last time. Due to the fast relative motion the curve of the scan points is displaced, rotated and curved when compared to the rear at time $t_{E}$ [cf. (b)]. The position of the rear for different times are indicated by parallel lines, the corresponding scan points by black dots.

Due to the temporal extent of the scanning process, relative motion between sensor and scanned car leads to blurring of the car's contour which will be referred to as motion-scan effect. This effect comprises shifting, dilating, rotating, and curving of the car's contour (cf. Fig. 1). The magnitude of this effect increases with decreasing scan frequency and increasing relative velocity. Only for very small distances and high relative velocities the curving is relevant. Estimating the parameters of a straight line on the basis of the lidar data leads to erroneous parameters for slope and intercept when the motion-scan effect is not taken into account. These can be critical when the distance is estimated too large. Errors of up to $0.5 \mathrm{~m}$ are possible under real conditions (cf. Section II-C).

The motion-scan effect depends on both the relative motion and the duration of the scanning process. Ballard and Vacherand [9] examined the effect of fast motion on the acquisition of range images. Blais et al. [10] dealt with the scanning of large objects with slow but significant motion of the scanner during the scanning process. Ono et al. [1] had a closer look at the effect of relative motion on the mapping of streets. The focus of their work was to compensate for the ego-motion on the basis of a sequence of lidar frames. Gräfe [11] also focused on the accurate mapping of streets. However, in contrast to Ono et al. he even considered relative motion during one scan by synchronizing every measured point to the estimated trajectory [best estimated trajectory (BET)]. Finally, there is extensive work on the estimation of poses and motion parameters of robots using a sequence of lidar frames, e.g., [12]. 
Our work-like Ballard and Vacherand-examines the effect of fast motion, but we focus on automotive applications. In contrast to Blais et al. we use a simple geometric model since the real data are corrupted by considerable measurement noise. Moreover, we use a simple motion model since a car's state does not change significantly during one scan frame. In contrast to Martínez et al. [12] the estimate of the car's pose requires only a single scan frame.

The objectives of this paper are as follows:

- quantifying the effect of fast motion on range images taken from cars (motion-scan effect);

- comparing this effect with statistical errors due to measurement noise;

- explicitly modeling the effect using simple models for both the geometry and the motion of the car;

- identifying the model parameters, which makes it possible to compensate for the motion-scan effect and to estimate the car's orientation using only a single scan frame.

An accurate reliable estimate of the car's position and orientation is crucial for safety-critical applications, e.g., driver assistance systems or autonomous vehicles.

The paper is organized as follows. First, the motion-scan effect is outlined in Section II. Then, Section III gives models for the rear, respectively, front and side of the car and the relative motion. Section IV examines which model parameters can be identified, while Section V shows how additional information can help to identify model parameters. Section VI explains how the motion-scan effect can be compensated for. The results are summarized in Section VII.

\section{OUtLining The PROBLEM}

In this section the effect of relative motion on range images is illustrated. First, assumptions valid throughout this paper are made. Next, the nomenclature is introduced. In the main part of this section the errors due to the motion-scan effect are quantified for the example of scanning a fast moving car. The section concludes with a comparison of these errors and statistical errors resulting from measurement noise.

\section{A. Assumptions}

All considerations are made under the following assumptions.

- Clustering of the lidar points is error-free.

- The straight line segments are ideal, i.e., no errors occur due to rounding of the car's corners.

- The measured data are free of noise.

- Measurements of distance and angle are not quantized.

- The measurement is time-discrete with constant sampling period $\Delta t$. Only in Section IV the measurement is assumed to be time-continuous. This guarantees that both ends of the straight line are detected, which is required when the width and the length of the scanned car are given as additional information.

- The sensor-car (index $s$ ) moves with constant known linear velocity $v_{s}$, while the scanned car moves with constant, but unknown linear velocity $v$ and constant angle $\psi$. Hence, the relative velocity is constant.

- The scanned car rolls without slippage. Thus, the instantaneous velocity vector is parallel to the longitudinal axis of the car.

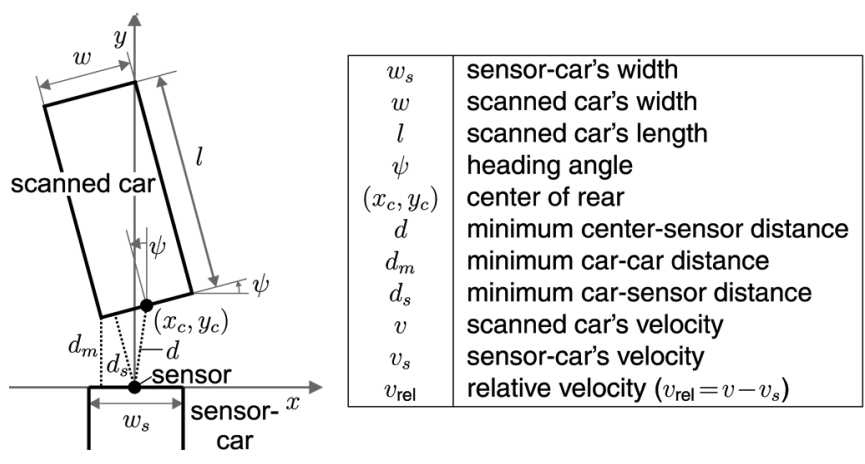

Fig. 2. Geometric relations between sensor-car and scanned car.

\section{B. Nomenclature}

The distance of two line segments (cf. Fig. 2) is usually characterized by the following.

- Distance $d$ of the centers. Since the sensor is positioned in the origin of the coordinate system, this simplifies to

$$
d=\left\|\left(\begin{array}{l}
x_{c} \\
y_{c}
\end{array}\right)\right\|_{2}
$$

- Minimum distance $d_{m}$ between two cars. The following is the shortest distance between two points, one located on the sensor-car and the other on the scanned car:

$$
d_{m}=\underset{\gamma \in\left[-\frac{w}{2}, \frac{w}{2}\right]}{\arg \min }\left\|\left(\begin{array}{l}
x_{c} \\
y_{c}
\end{array}\right)+\gamma\left(\begin{array}{c}
\cos \psi \\
\sin \psi
\end{array}\right)-\lambda\left(\begin{array}{l}
1 \\
0
\end{array}\right)\right\|_{2}
$$

Width $w$ must be known. Equation (2) is either the orthogonal projection of one corner onto the other line segment or the minimum distance between two corners.

- Minimum distance $d_{s}$ between sensor and car. This can be obtained from (2) by setting $\lambda=0$, since the sensor is fixed in the center of the line segment.

Distance $d$ is easy to compute, but it does not show how close the two cars really are. In contrast to this, the minimum distance $d_{m}$ cannot be calculated in a straightforward way. Moreover, it requires that width $w$ is known.

The relationship between the distances $d, d_{m}$, and $d_{s}$ is given by

$$
d_{m} \leq d_{s} \leq d \leq d_{s}+\frac{w}{2} \leq d_{m}+\frac{w+w_{s}}{2} .
$$

It has already been pointed out in Section I that pose and orientation of a moving line segment can only be indicated using a reference point in time. There are five significant reference points in time for lidar scanners (cf. Fig. 3). The transformation of the line segment parameters for different points in time is given in Section III.

\section{Simulation Results}

1) Simulation Settings: Distance $d$ and reference point in time $t_{F}$ are used for the simulation of the motion-scan effect. The simulation allows the motion-scan effect to be examined exclusively without any disturbing effects due to measurement errors, quantization, etc. The parameters used for the simulation 


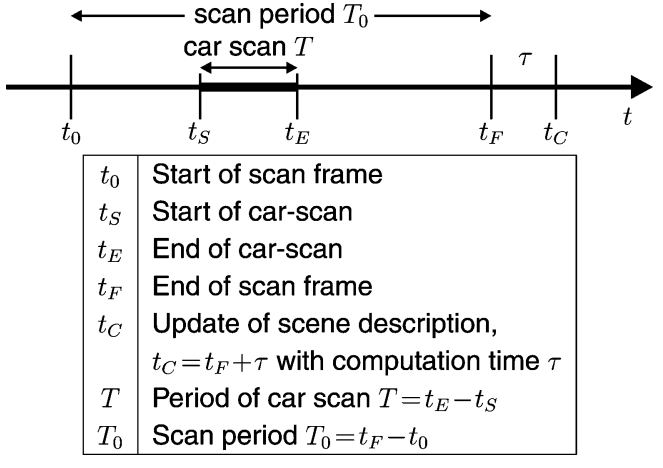

Fig. 3. Time bar showing the reference points in time.

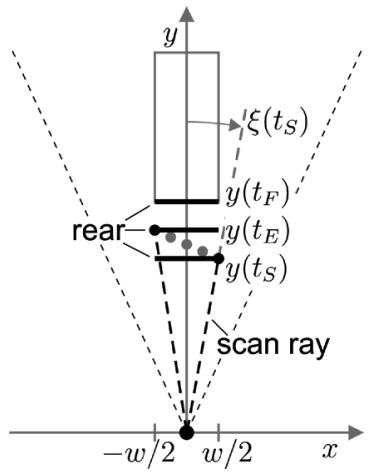

Fig. 4. Scanning of the car's rear starts at time $t_{S}$ [scan angle $\left.\xi\left(t_{S}\right)\right]$ and ends at $t_{E}$. The scan frame ends at $t_{F}$. Scan points are indicated by dots.

are close to real-world situations, which leads to reliable quantitative simulation results. For this a typical medium-class car is scanned by a state-of-the-art lidar scanner.

- Car (typical medium-class car):

— width $w=1.70 \mathrm{~m}$;

- length $l=4.20 \mathrm{~m}$.

- Lidar scanner:

- angle range $\xi \in\left[-20^{\circ}, 20^{\circ}\right]$ (between positive $y$ axis and scan ray);

— angular resolution $\Delta \xi=0.1^{\circ}$;

- scan frequency $f_{0}=10 \mathrm{~Hz}$ corresponding to a scan period of $T_{0}=0.1 \mathrm{~s}$ (per frame).

2) Simulation of the Motion-Scan Effect: In Section I the consequences of the motion-scan effect have been described. In this section the consequences of the effect will be quantified using simulation runs. For this two situations are examined.

1) The sensor-car follows the scanned car (cf. Fig. 4). Both cars move along the same straight line with constant positive but different velocities. Large positive values for $v_{\text {rel }}$ occur after lane-changing maneuvers, when the sensor-car has to stop abruptly or when it is being overtaken. However, especially critical are large negative velocities $v_{\text {rel }}$, for instance when the scanned car breaks sharply. The results are given in Table I. Their calculation is explained in Appendix I.

2) The scanned car is approaching on the neighboring lane (cross-distance of the cars' centers: $3.2 \mathrm{~m}$ ). In this situation large relative velocities of more than $50 \mathrm{~m} / \mathrm{s}=180 \mathrm{~km} / \mathrm{h}$
TABLE I

ERrors Due to Relative Motion When BotH CARS ARE Driving IN the SAME Lane

\begin{tabular}{|r|r|r|r|r|}
\hline$v_{\text {rel }}$ in $\mathrm{m} / \mathrm{s}$ & $d_{t_{F}}$ in $\mathrm{m}$ & $\Delta \tilde{d}$ in $\mathrm{m}$ & $\Delta \widetilde{\psi}$ in ${ }^{\circ}$ & $\Delta \widetilde{w}$ in $\mathrm{m}$ \\
\hline \multicolumn{5}{|c|}{ The cars are driving at a constant distance. } \\
0.00 & 5.00 & 0.00 & 0.00 & 0.00 \\
0.00 & 10.00 & 0.00 & 0.00 & 0.00 \\
0.00 & 20.00 & 0.00 & 0.00 & 0.00 \\
\hline \multicolumn{6}{|c|}{ The scanned car is receding. } \\
5.00 & 5.00 & -0.03 & -0.91 & 0.00 \\
10.00 & 5.00 & -0.06 & -1.83 & 0.00 \\
5.00 & 10.00 & -0.03 & -0.46 & 0.00 \\
10.00 & 10.00 & -0.06 & -0.92 & 0.00 \\
\hline \multicolumn{5}{|c|}{ The scanned car is approaching. } \\
-5.00 & 5.00 & 0.03 & 0.90 & 0.00 \\
-10.00 & 5.00 & 0.06 & 1.79 & 0.00 \\
-5.00 & 10.00 & 0.03 & 0.45 & 0.00 \\
-10.00 & 10.00 & 0.06 & 0.91 & 0.00 \\
-5.00 & 20.00 & 0.03 & 0.23 & 0.00 \\
-10.00 & 20.00 & 0.06 & 0.45 & 0.00 \\
\hline
\end{tabular}

TABLE II

ERrors Due to Relative Motion WhEN THE SCANNED CAR Is APPROACHING IN THE NEIGHBORING LANE

\begin{tabular}{|c|c|r|r|c|}
\hline$v_{\text {rel }}$ in $\mathrm{m} / \mathrm{s}$ & $d_{t_{F}}$ in $\mathrm{m}$ & $\Delta \tilde{d}$ in $\mathrm{m}$ & $\Delta \widetilde{\psi}$ in $^{\circ}$ & $\Delta \widetilde{w}$ in $\mathrm{m}$ \\
\hline-5.00 & 20.00 & 0.02 & 0.22 & 0.00 \\
-10.00 & 20.00 & 0.03 & 0.44 & 0.00 \\
-15.00 & 20.00 & 0.05 & 0.67 & 0.00 \\
-20.00 & 20.00 & 0.06 & 0.89 & 0.00 \\
-30.00 & 20.00 & 0.09 & 1.33 & 0.00 \\
-40.00 & 20.00 & 0.12 & 1.78 & 0.00 \\
-50.00 & 20.00 & 0.15 & 2.22 & 0.00 \\
\hline
\end{tabular}

are possible on country roads. Here, distances are measured with respect to the front of the scanned car. The results are given in Table II. Their calculation is explained in Appendix II.

For the situations the true distance $d_{t_{F}}$ at the end of the scan frame, i.e., $t_{F}$, is given. First, an ordinary least squares (LS) approach is used to fit a line segment to the scan points. The distance of its center to the $x$ axis of the coordinate system yields the computed distance $\tilde{d}$. The tilt $\Delta \tilde{\psi}$ of the scanned car w.r.t. its orientation in the sensor coordinate system is directly given by the slope of the line segment since $\psi_{t_{F}}=\psi=0$ holds for both cases. The distance between first and last scan point gives an estimate $\tilde{w}$ for the width of the scanned car.

Evaluation of the Simulation Results:

- When $d_{t_{F}}$ and $v_{\text {rel }}$ are given, distance $d$ can be computed for any point in time, e.g., for $t=t_{S}: d_{t_{S}}=d_{t_{F}}-v_{\text {rel }}\left(t_{F}-\right.$ $\left.t_{S}\right)$.

- The period of time while the car is scanned, i.e., $T=t_{E}-$ $t_{S}$, increases with increasing width $w$, decreasing distance $d_{t_{F}}$, and decreasing relative velocity $v_{\text {rel }}$.

- Tables I and II show that the distance error $\Delta \tilde{d}=\tilde{d}-d_{t_{F}}$ can be significant and exceed $0.1 \mathrm{~m}$. 
TABLE III

LS Estimate of the Line SEgment Parameters IN NOISY LIDAR DATA WITH $v_{\text {rel }}=0$ AND $\sigma_{R}=0.10 \mathrm{~m}$

\begin{tabular}{|r|c|c|r|}
\hline$d$ in $\mathrm{m}$ & $\Delta \hat{d}$ in $\mathrm{m}$ & $\Delta \hat{\psi}$ in $^{\circ}$ & \multicolumn{1}{|c|}{$N$} \\
\hline 5.00 & \pm 0.02 & \pm 2.10 & 193 \\
10.00 & \pm 0.03 & \pm 2.92 & 97 \\
20.00 & \pm 0.03 & \pm 4.17 & 49 \\
\hline
\end{tabular}

- When the sensor-car is following the scanned car and their relative velocity $v_{\text {rel }}<0$, the distance is estimated too large. This result is dangerous since the real distance is closer than it seems to be. When the scanned car is approaching in a neighboring lane, the distance is also estimated too large.

- The tilt $\Delta \tilde{\psi}=\tilde{\psi}$ is less than $2.3^{\circ}$. The dilation error $\Delta \tilde{w}=$ $\tilde{w}-w$ can be neglected.

- Within the simulation the case of constant deceleration has also been examined, e.g., when braking with half the acceleration of gravity $g$. The additive effect is very small since $T$ is very small. In the following we give the results for $v_{\text {rel }, 0}=5 \mathrm{~m} / \mathrm{s}, d_{t_{F}}=10 \mathrm{~m}$ and $\dot{v}=-0.5 g: \Delta \tilde{d}=-0.03$ $\mathrm{m}, \Delta \tilde{\psi}=-0 / 45^{\circ}, \Delta \tilde{w}=0.00 \mathrm{~m}$. Comparing these results with the corresponding row of Table I (third row of second block) shows that braking even mitigates the motion-scan effect since the relative motion decreases during the scan.

- High relative velocities occur when the scanned car is approaching in the neighboring lane or when the sensor-car is approaching a parked car.

3) Comparison With Statistical Errors: Errors due to relative motion during the scan are compared with errors resulting from measurement errors. For the statistical examination a line segment with $v_{\text {rel }}=0$ is scanned. Thus there are no additional errors due to relative motion and the distance $d$ is constant, i.e., $d=d_{t_{F}}=$ const. The angle measurement is assumed to be noise-free while the measurement noise of the distance is modeled as independent Gaussian $\mathcal{N}\left(0 ; \sigma_{R}^{2}\right)$ with standard deviation $\sigma_{R}=0.10 \mathrm{~m}$. Details to the statistical examination can be found in [13].

In the symmetric case, when the line segment is symmetric to the positive $y$ axis, the estimation of the slope is unbiased and the estimation of the distance is asymptotically unbiased. Therefore, only the standard deviations are of major interest for this special case. For this special case the impact of measurement errors on the estimation of a line are examined. For this simulation a horizontal line segment of length $w=1.70 \mathrm{~m}$ was scanned for different distances $d$. The radial component of the ideal data was corrupted by additive Gaussian noise. LS estimates of distance $d$ and heading angle $\psi$ were determined. This procedure was repeated 10000 times to obtain an estimate of the corresponding standard deviations. The 2.5- $\sigma$ confidence intervals $\Delta \hat{d}$ and $\Delta \hat{\psi}$ for different distances $d$ are given in Table III. $N$ denotes the number of scan points.

Typically, the error of the LS estimate $\hat{d}$ is much smaller than $\sigma_{R}$. This holds since the estimate is supported by many measurement points and the noise process is assumed to be uncorrelated. Thus, the standard deviation of the LS estimate is approximately proportional to the inverse of $\sqrt{N}$. With the distance increasing,

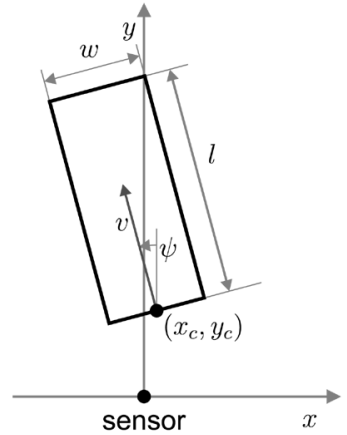

(a)

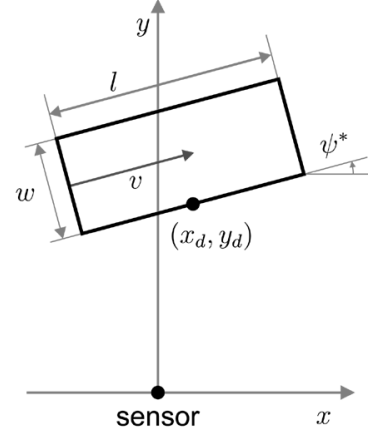

(b)
Fig. 5. Dimensions of the scanned car. In Fig. 5(a) the rear, respectively, front of the car is visible, in Fig. 5(b) its side. (a) Rear/front of the car. (b) Side of the car.

the effect of noise on the single measurement decreases. However, the number of scan points $N$ decreases as well, which affects the estimate more severely and overall leads to worse results.

The distance and orientation error due to relative motion exceed the errors resulting from measurement noise. For the relative velocity $v_{\text {rel }}=-10 \mathrm{~m} / \mathrm{s}$ and the distance $d_{t_{F}}=d=10 \mathrm{~m}$ the deterministic errors are $\Delta \tilde{d}=0.06 \mathrm{~m}$ and $\Delta \tilde{\psi}=0.91^{\circ}$ compared to $\pm 0.03 \mathrm{~m}$ and $\pm 2.92^{\circ}$ with a confidence of $98 \%$.

To sum up, the effects due to relative motion can be in the magnitude of statistical errors. Therefore, they are of practical importance and subject to a close examination taken out in the following sections.

\section{Modeling A Moving CAR's CONTOUR USING TIME-VARIANT LiNE SEgMENTS}

Section II quantified the motion-scan effect on the basis of a simulation. The results revealed that this effect is of practical importance. Therefore, simple models for the moving contour of a car are given in this section. Consecutively, the model parameters are checked for identifiability in Section IV. Identification of the model parameters makes it possible to compensate for the negative impacts of the motion-scan effect.

In this section a moving car's contour is modeled using timevariant line segments. Each of these line segments is characterized by its center's position $\left(x_{c}, y_{c}\right)$, its heading angle $\psi$ and its width $w$ or its length $l$ (cf. Fig. 5). Due to the relative motion the line segment's parameters become time-variant and depend both on the velocities $v$ and $v_{s}$ and the reference time $t_{F}$. The velocity $v_{s}$ of the sensor-car is assumed to be known, e.g., it is measured by an odometer. This time-variant model makes it possible to use scan points taken at different times to identify the model parameters. Moreover, this model is formulated such that the scan points' coordinates can be used directly.

In Section III-A the line segment model for the front and rear is derived and in Section III-B the model for the side.

\section{A. Line Segment Model of the Rear, Respectively, Front}

Since the period while the car is scanned, i.e., $T=t_{E}-t_{S}$, is rather short, the relative motion of sensor-car and scanned car can be approximated by a linear motion for typical traffic situations, i.e., velocity $v$ and heading angle $\psi$ are constant. Hence, 
the position of the rear's center is given by

$$
\begin{aligned}
& x_{c}(t)=x_{c . t_{S}}-v \sin \psi\left(t-t_{S}\right) \\
& y_{c}(t)=y_{c . t_{S}}+\left(v \cos \psi-v_{s}\right)\left(t-t_{S}\right)
\end{aligned}
$$

where $\left(x_{c . t_{S}}, y_{c . t_{S}}\right)$ denotes the center's position at time $t_{S}$.

Even though the parameters for time $t_{S}$ are estimated, the parameters for time $t_{F}$ are relevant, since they give the scanned car's position at the end of the scan frame. Using a simple parameter transformation following (3), the practically relevant parameters at time $t_{F}$ can be obtained from those at time $t_{S}$

$$
\begin{aligned}
& x_{c . t_{F}}:=x_{c}\left(t_{F}\right)=x_{c . t_{S}}-v \sin \psi\left(t_{F}-t_{S}\right) \\
& y_{c . t_{F}}:=y_{c}\left(t_{F}\right)=y_{c . t_{S}}+\left(v \cos \psi-v_{s}\right)\left(t_{F}-t_{S}\right) .
\end{aligned}
$$

This transformation predicts the position of the car depending on the model parameters. Thus, it is also possible to take into account additional computation time $\tau$ by replacing $t_{F}$ by $t_{C}$ in (4) (cf. Fig. 3).

The line segment is parametrized using a time-variant line model with fixed slope $m$ given by

$$
y(t)=m x(t)+c(t) .
$$

The problem when identifying line model $\left(m, c_{i}\right)$ at time $t_{i}$ is that there is only one single scan point $\left(x_{i}, y_{i}\right)$. Therefore, the line model has to be constructed such that $c(t)$ is expressed in terms of time-invariant parameters which is explained in Appendix III. With this expression for $c(t)(5)$ can be written as

$$
y=\tan \psi\left(x-x_{c . t_{F}}\right)+y_{c . t_{F}}+\left(\frac{v}{\cos \psi}-v_{s}\right)\left(t-t_{F}\right) .
$$

Thus, several points $\left(x_{i}, y_{i}\right)$ taken at different points in time can be used for the estimation of the time-invariant parameters.

In order to derive a line segment model from a line model, the width $w$ has to be restricted. Then the line segment for a point in time $t_{i}$ is given by all points $\left(x_{i}, y_{i}\right)$ fulfilling $(6 \mathrm{a})$ and

$$
\left\|\left(\begin{array}{l}
x_{i} \\
y_{i}
\end{array}\right)-\left(\begin{array}{c}
x_{c . t_{F}}-v \sin \psi\left(t_{i}-t_{F}\right) \\
y_{c . t_{F}}+\left(v \cos \psi-v_{s}\right)\left(t_{i}-t_{F}\right)
\end{array}\right)\right\|_{2} \leq \frac{w}{2} .
$$

When both corners are visible, the parameter $w$ of the line segment can be determined by

$$
w=\left\|\left(\begin{array}{l}
x\left(t_{E}\right) \\
y\left(t_{E}\right)
\end{array}\right)-\left(\begin{array}{c}
x\left(t_{S}\right)-v \sin \psi\left(t_{E}-t_{S}\right) \\
y\left(t_{S}\right)+\left(v \cos \psi-v_{s}\right)\left(t_{E}-t_{S}\right)
\end{array}\right)\right\|_{2} .
$$

\section{B. Line Segment Model of the Side}

The formulas for the line segment model of the side can be derived analogously to the formulas of the rear. The derivation given in Appendix IV yields the line model

$$
y=\tan \psi^{*}\left(x-x_{d . t_{F}}\right)+y_{d . t_{F}}-v_{s}\left(t-t_{F}\right)
$$

with the restriction

$$
\left\|\left(\begin{array}{c}
x_{i} \\
y_{i}
\end{array}\right)-\left(\begin{array}{c}
x_{d . t_{F}}+v \cos \psi^{*}\left(t_{i}-t_{F}\right) \\
y_{d . t_{F}}+\left(v \sin \psi^{*}-v_{s}\right)\left(t_{i}-t_{F}\right)
\end{array}\right)\right\|_{2} \leq \frac{l}{2} .
$$

When both corners are visible, the parameter $l$ is given by

$$
l=\left\|\left(\begin{array}{l}
x\left(t_{E}\right) \\
y\left(t_{E}\right)
\end{array}\right)-\left(\begin{array}{c}
x\left(t_{S}\right)+v \cos \psi^{*}\left(t_{E}-t_{S}\right) \\
y\left(t_{S}\right)+\left(v \sin \psi^{*}-v_{s}\right)\left(t_{E}-t_{S}\right)
\end{array}\right)\right\|_{2} .
$$

\section{Conclusion of this Section}

Equations (6a) and (7a) fulfill the requirements given at the beginning of this section. In particular, $x$ and $y$ can directly be replaced by the scan points $\left(x_{i}, y_{i}\right)$ which results in one equation for each scan point. However, it has to be checked if the model parameters can be identified using these equations. Identifiability cannot be deduced from standard approaches using time-invariant line segment models, e.g., [14] and [15], since the time-variant models given in (3) and (7) have the additional parameter $v$. Therefore, the following section examines the identifiability of the model parameters.

\section{IDENTIFIABILITy OF THE Model Parameters}

Section IV-A examines which parameters of line segment model (6) and Section IV-B which parameters of model (7) can be identified given a set of scan points.

\section{A. Identifiability for Rear and Front End}

Model (6) has the five free parameters $\psi, v, x_{c . t_{S}}, y_{c . t_{S}}$ and $w$. Here $t_{S}$ is chosen as reference point in time, and parameters $x_{c}$ and $y_{c}$ are transformed using (4)

$$
\begin{aligned}
& y=\tan \psi\left(x-x_{c . t_{S}}\right)+y_{c . t_{S}}+\left(\frac{v}{\cos \psi}-v_{s}\right)\left(t-t_{S}\right) \\
& w=\left\|\left(\begin{array}{l}
x\left(t_{E}\right) \\
y\left(t_{E}\right)
\end{array}\right)-\left(\begin{array}{c}
x\left(t_{S}\right)-v \sin \psi\left(t_{E}-t_{S}\right) \\
y\left(t_{S}\right)+\left(v \cos \psi-v_{s}\right)\left(t_{E}-t_{S}\right)
\end{array}\right)\right\|_{2} .
\end{aligned}
$$

In order to check this problem for identifiability, (8a) is written as a linear equation system

$$
\underbrace{\left(\begin{array}{lll}
1 & x_{1} & t_{1}-t_{S} \\
1 & x_{2} & t_{2}-t_{S} \\
1 & x_{3} & t_{3}-t_{S}
\end{array}\right)}_{=: H} \cdot\left(\begin{array}{l}
\theta_{1} \\
\theta_{2} \\
\theta_{3}
\end{array}\right)=\left(\begin{array}{l}
y_{1}+v_{s}\left(t_{1}-t_{S}\right) \\
y_{2}+v_{s}\left(t_{2}-t_{S}\right) \\
y_{3}+v_{s}\left(t_{3}-t_{S}\right)
\end{array}\right)
$$

with the parameters

$$
\begin{aligned}
& \theta_{1}=-x_{c . t_{S}} \tan \psi+y_{c . t_{S}} \\
& \theta_{2}=\tan \psi \\
& \theta_{3}=\frac{v}{\cos \psi} .
\end{aligned}
$$

Matrix $H$ is regular when the vectors $1_{3}, x$, and $t-t_{S}$ are linearly independent which usually is the case. Consequently, the three parameters $\theta_{i}$ can be identified. At the most three model parameters can be estimated with the $\theta_{i}$.

Equation (10b) shows that $\psi$ can be identified. Equation (10c) together with the already determined parameter $\psi$ can be used to obtain $v$ since the sensor-car's velocity $v_{s}$ is assumed to be known. However, the ambiguity between $x_{c . t_{S}}$ 
and $y_{c . t_{S}}$ as given in (10a) cannot be resolved. Equation $x_{c . t_{S}} \tan \psi+y_{c . t_{S}}=$ const describes a line in the $x-y$ plane. This means that the position of the center on this line is not fixed without any additional information, e.g., the width $w$.

Equation (8b) shows that width $w$ can be identified when both corners, $\left(x\left(t_{S}\right), y\left(t_{S}\right)\right)$ and $\left(x\left(t_{E}\right), y\left(t_{E}\right)\right)$, are visible. When width $w$, velocity $v$, and angle $\psi$ are known, the position of the center is given by

$\left(\begin{array}{l}x_{c . t_{S}} \\ y_{c . t_{S}}\end{array}\right)=0.5\left(\begin{array}{c}x\left(t_{S}\right)+x\left(t_{E}\right)-v \sin \psi\left(t_{S}-t_{E}\right) \\ y\left(t_{S}\right)+y\left(t_{E}\right)+\left(v \cos \psi-v_{s}\right)\left(t_{S}-t_{E}\right)\end{array}\right)$.

\section{B. Identifiability for Side}

The model of the side given by (7) has five free parameters, $\psi^{*}, v, x_{d . t_{S}}, y_{d . t_{S}}$, and $l$

$$
\begin{aligned}
y & =\tan \psi^{*}\left(x-x_{d . t_{S}}\right)+y_{d . t_{S}}-v_{s}\left(t-t_{S}\right) \\
l & =\left\|\left(\begin{array}{c}
x\left(t_{E}\right) \\
y\left(t_{E}\right)
\end{array}\right)-\left(\begin{array}{c}
x\left(t_{S}\right)+v \cos \psi^{*}\left(t_{E}-t_{S}\right) \\
y\left(t_{S}\right)+\left(v \sin \psi^{*}-v_{s}\right)\left(t_{E}-t_{S}\right)
\end{array}\right)\right\|_{2} .
\end{aligned}
$$

In order to examine the identifiability, (12a) is written as a linear equation system

$$
\underbrace{\left(\begin{array}{ll}
1 & x_{1} \\
1 & x_{2}
\end{array}\right)}_{=: H} \cdot\left(\begin{array}{l}
\theta_{1} \\
\theta_{2}
\end{array}\right)=\left(\begin{array}{l}
y_{1}+v_{s}\left(t_{1}-t_{S}\right) \\
y_{2}+v_{s}\left(t_{2}-t_{S}\right)
\end{array}\right)
$$

with the parameters

$$
\begin{aligned}
& \theta_{1}=-x_{d . t_{S}} \tan \psi^{*}+y_{d . t_{S}} \\
& \theta_{2}=\tan \psi^{*} .
\end{aligned}
$$

Matrix $H$ is always regular. Since parameter vector $\theta$ has only two elements, two parameters can be estimated at most. Since the velocity $v$ of the scanned car is not contained in (12a), it cannot be estimated without any additional information. This is also obvious since all measurement points are elements of one single line when scanning the side. This line is parallel to the vector of relative velocity $v-v_{s}$. Even when both front and rear, i.e., start-and endpoint of the line segment of the car, are scanned, it is not possible to distinguish between a slow-driving short and a fast-driving long car.

Equation (14b) shows that $\psi^{*}$ can be identified. However, in contrast to (11) it is not possible to estimate the center of the line segment since $v$ cannot be identified.

\section{AdDitional Information in Automotive APPLiCATiOns}

Section IV showed that it is not always possible to identify all model parameters given a set of scan points, e.g., when scanning the side the velocity $v$ of the car cannot be determined. Therefore, additional information is required to make all model parameters identifiable which makes it possible to compensate for the motion-scan effect. This section gives examples of practically relevant additional information. Additional information is not only important for the identifiability, but it also improves and simplifies the parameter estimation in most cases.

In the following, three kinds of practically relevant additional information are examined. Unless stated otherwise, both corners of the scanned car are visible.
TABLE IV

LEGEND TO TABLES V AND VI

\begin{tabular}{|c|l|}
\hline$F$ & closely following at short distance, $m=0$ \\
$L$ & width $w$ or length $l$ of car is known \\
$C$ & cognitive driving: absolute velocity $v$ is known \\
$N$ & min. number of points required for identification \\
$\checkmark$ & parameter can be identified \\
$\times$ & parameter is given \\
+ & additional information is given \\
- & additional information is not given \\
$\dagger$ & at least one corner visible \\
$\ddagger$ & both corners visible \\
\hline
\end{tabular}

TABLE V

IDENTIFIABILITY USING ADDITIONAL INFORMATION WHEN THE REAR OR FRONT OF THE CAR IS VISIBLE

\begin{tabular}{|c|c|c|c|c|c|c|c|c|}
\hline$F$ & $L$ & $C$ & $N$ & $\psi$ & $v$ & $x_{c . t_{S}}$ & $y_{c . t_{S}}$ & $w$ \\
\hline \hline+ & - & - & $\geqslant 2^{\ddagger}$ & $=0$ & $\checkmark$ & $\checkmark$ & $\checkmark$ & $\checkmark$ \\
\hline+ & + & - & $\geqslant 2^{\dagger}$ & $=0$ & $\checkmark$ & $\checkmark$ & $\checkmark$ & $\times$ \\
\hline+ & - & + & $\geqslant 2^{\ddagger}$ & $=0$ & $\times$ & $\checkmark$ & $\checkmark$ & $\checkmark$ \\
\hline+ & + & + & $\geqslant 1^{\dagger}$ & $=0$ & $\times$ & $\checkmark$ & $\checkmark$ & $\times$ \\
\hline- & - & - & $\geqslant 3^{\ddagger}$ & $\checkmark$ & $\checkmark$ & $\checkmark$ & $\checkmark$ & $\checkmark$ \\
\hline- & + & - & $\geqslant 3^{\dagger}$ & $\checkmark$ & $\checkmark$ & $\checkmark$ & $\checkmark$ & $\times$ \\
\hline- & - & + & $\geqslant 3^{\ddagger}$ & $\checkmark$ & $\times$ & $\checkmark$ & $\checkmark$ & $\checkmark$ \\
\hline- & + & + & $\geqslant 2^{\dagger}$ & $\checkmark$ & $\times$ & $\checkmark$ & $\checkmark$ & $\times$ \\
\hline
\end{tabular}

TABLE VI

IDENTIFIABILITY USING ADDITIONAL INFORMATION WHEN THE SIDE OF THE CAR IS VISIBLE

\begin{tabular}{|c|c|c|c|c|c|c|c|}
\hline$L$ & $C$ & $N$ & $\psi^{*}$ & $v$ & $x_{d . t_{S}}$ & $y_{d . t_{S}}$ & $l$ \\
\hline \hline- & - & $\geqslant 2 \ddagger$ & $\checkmark$ & - & \multicolumn{4}{|c|}{$f\left(x_{\text {d.t. }}, y_{d . t_{S}}\right)=0$} & - \\
\hline+ & - & $\geqslant 2^{\ddagger}$ & $\checkmark$ & $\checkmark$ & $\checkmark$ & $\checkmark$ & $\times$ \\
\hline- & + & $\geqslant 2^{\ddagger}$ & $\checkmark$ & $\times$ & $\checkmark$ & $\checkmark$ & $\checkmark$ \\
\hline+ & + & $\geqslant 2^{\dagger}$ & $\checkmark$ & $\times$ & $\checkmark$ & $\checkmark$ & $\times$ \\
\hline
\end{tabular}

1) The sensor-car follows the scanned car at a short distance, i.e., $v_{x}=0$ and $m=0$. Slope $m=0$ is also possible when the car is approaching in a neighboring lane. This kind of additional information is denoted by $F$ in Tables $\mathrm{V}$ and VI.

2) In the case of cognitive driving the cars can communicate with each other and can exchange information. For the situation considered here the velocity of the scanned car, i.e., $v$, is of particular interest. This kind of additional information is denoted by $C$ and includes the case $v=0$.

3) Sometimes the dimensions of the other car, i.e., its width $w$ or its length $l$, are known (cf. Fig. 5). This information 
could be the result of a classification, or it could be transmitted from a cognitive car. This kind of additional information is denoted by $L$ and requires that at least one corner of the contour is scanned.

\section{A. Additional Information for Rear and Front End}

The line segment is described by (6). Section IV-A showed that all parameters can be identified when both corners are visible. However, additional information is required when only one corner is visible. Moreover, it improves parameter estimation.

- When the width $w$ of the car is known and only its right corner is within the field of view, $\left(x_{c . t_{S}}, y_{c . t_{S}}\right)$ can be determined

$$
\left(\begin{array}{l}
x_{c . t_{S}} \\
y_{c . t_{S}}
\end{array}\right)=\left(\begin{array}{l}
x\left(t_{S}\right) \\
y\left(t_{S}\right)
\end{array}\right)-\frac{w}{2}\left(\begin{array}{c}
\cos \psi \\
\sin \psi
\end{array}\right) \text {. }
$$

The corresponding holds when only the left corner can be seen

$$
\begin{aligned}
\left(\begin{array}{l}
x_{c . t_{S}} \\
y_{c . t_{S}}
\end{array}\right)= & \left(\begin{array}{l}
x\left(t_{E}\right) \\
y\left(t_{E}\right)
\end{array}\right)+\frac{w}{2}\left(\begin{array}{c}
\cos \psi \\
\sin \psi
\end{array}\right) \\
& +\left(\begin{array}{c}
-v \sin \psi \\
v \cos \psi-v_{s}
\end{array}\right)\left(t_{S}-t_{E}\right) .
\end{aligned}
$$

- A cognitive car in front transmitting its own velocity $v$ does not provide any additional information, since $v$ is already known.

- Following the car closely with $\psi=0$ allows to identify width $w$

$$
w=\left|x\left(t_{E}\right)-x\left(t_{S}\right)\right|
$$

Any additional information, even when it is not necessary to identify the model parameters, can be used to support the estimation of the parameters.

The results of this subsection are summarized in Table V. The legend to Tables V and VI is given in Table IV.

\section{B. Additional Information for the Side}

Equation (12) describes the line segment model of the side. It has already been pointed out in Section IV-B, that parameter $\psi^{*}$ can be identified whereas there remains an ambiguity between $x_{d . t_{S}}$ and $y_{d . t_{S}}$. This ambiguity is denoted by $f(\cdot)=0$ in Table VI. Moreover, neither velocity $v$ nor length $l$ can be identified without additional information.

- When the length $l$ of the scanned car is known and both corners are visible, the center $\left(x_{d . t_{S}}, y_{d . t_{S}}\right)$ can be identified

$$
\begin{aligned}
& \left(\begin{array}{l}
x_{d . t_{S}} \\
y_{d . t_{S}}
\end{array}\right)=\left(\begin{array}{l}
x\left(t_{S}\right) \\
y\left(t_{S}\right)
\end{array}\right)-\frac{l}{2}\left(\begin{array}{c}
\cos \psi^{*} \\
\sin \psi^{*}
\end{array}\right) \\
& \left(\begin{array}{l}
x_{d . t_{E}} \\
y_{d . t_{E}}
\end{array}\right)=\left(\begin{array}{l}
x\left(t_{E}\right) \\
y\left(t_{E}\right)
\end{array}\right)+\frac{l}{2}\left(\begin{array}{c}
\cos \psi^{*} \\
\sin \psi^{*}
\end{array}\right) .
\end{aligned}
$$

With this information the car's velocity $v$ can be identified

$$
v=\frac{1}{t_{E}-t_{S}}\left\|\left(\begin{array}{c}
x_{d . t_{E}} \\
y_{d . t_{E}}-v_{s}\left(t_{E}-t_{S}\right)
\end{array}\right)-\left(\begin{array}{l}
x_{d . t_{S}} \\
y_{d . t_{S}}
\end{array}\right)\right\|_{2} \cdot
$$

- When the velocity $v$ of the scanned car is known and both corners are visible, first the length $l$ is determined using (12b) and then its center.

The results of this subsection are summarized in Table VI.

Section V showed that all model parameters can be identified given appropriate additional information. When scanning the rear additional information is not necessary when both corners are visible, but it helps to improve the parameter estimation. When scanning the side only additional information about the length $l$ makes all model parameters identifiable. Identification of the model parameters is important since it is required for compensation of the motion-scan effect.

\section{COMPENSATION FOR THE MOTION-SCAN EFFECT}

Section IV examined which model parameters can be identified given a set of scan points. For the cases when not all parameters can be identified Section $\mathrm{V}$ showed how additional information can be exploited to identify model parameters. This section explains how the model parameters can be determined and how the true motion parameters can be obtained. Thus, it is possible to compensate for the motion-scan effect.

\section{A. Rear and Front Model}

Given a set of $N \geqslant 3$ scan points with both corners being visible an overdetermined linear equation system is formulated that extends (9) to $N$ scan points. The three parameters $\theta_{i}$ can be computed using an LS approach. The heading angle $\psi$ is given by (10b): $\psi=\arctan \theta_{2}$. The result together with (10c) yields $v: v=\theta_{3} \cos \psi$. With $\psi$ and $v$ being known $\left(x_{c . t_{S}}, y_{c . t_{S}}\right)$ is determined using (11). From this $\left(x_{c . t_{F}}, y_{c . t_{F}}\right)$ is obtained using (4). With $\left(x_{c . t_{F}}, y_{c . t_{F}}\right)$ the distance at the end of the frame is given by $d_{c . t_{F}}=\left\|\left(x_{c . t_{F}}, y_{c . t_{F}}\right)^{\mathrm{T}}\right\|_{2}$ where $(\cdot)^{\mathrm{T}}$ denotes matrix transpose. When necessary the width $w$ can be computed using (6c).

Hence the velocity $v$, the heading angle $\psi$, the distance $d_{c . t_{F}}$ at the end of the scan frame and the width of the car can be recovered without any errors due to the motion-scan effect.

\section{B. Side Model}

As Section IV-B indicated additional information is required to identify the model parameters. This subsection shows how the true motion parameters can be recovered given either the length $l$ or the velocity $v$. At first (13) is extended to an overdetermined linear equation system. The parameters $\theta_{i}$ are computed using an LS approach. With (14b) the heading angle $\psi^{*}$ is given by $\psi^{*}=\arctan \theta_{2}$.

If the length $l$ is given and both corners are visible $\left(x_{d . t_{S}}, y_{d . t_{S}}\right)$ and $\left(x_{d . t_{E}}, y_{d . t_{E}}\right)$ can be computed with (17). Putting these results in (18) gives the velocity $v$. Then the position of the center at the end of the scan frame can be determined using (4). Finally, the true distance $d_{d . t_{F}}$ at the end of the scan frame can be computed with $d_{d . t_{F}}=\left\|\left(x_{d . t_{F}}, y_{d . t_{F}}\right)^{\mathrm{T}}\right\|_{2}$.

If the velocity $v$ is given and both corners are visible, the length $l$ is determined by (12b).

Since the length $l$ is known, one can proceed like in the previous case. 
Hence, the motion parameters can be recovered without any errors due to the motion-scan effect.

\section{SUMMARY}

This paper examines the motion-scan effect for lidar scanners that are used in cars. This effect occurs when a lidar sensor scans a car that moves with fast velocity relatively to the sensor-car. After a short description of the effect the errors due to this effect are quantified using simulation runs. The errors are compared to statistical errors due to measurement noise. Both errors are of the same magnitude. The measurement noise can be reduced by better sensors whereas the motion-scan effect can be reduced by a faster scan.

In order to compensate for the motion-scan effect simple models of the scanned car's contour and the relative motion are formulated. The model parameters are checked for identifiability. While all parameters can be identified when scanning a car's rear, only the heading angle can be identified when scanning the side. Therefore, practically relevant additional information, e.g., the scanned car's velocity, is considered. Thus, all model parameters are identifiable in many cases and compensation of the motion-scan effect is possible. Moveover, the model parameters can be identified on the basis of a single scan frame. This paper adds to the authors' examination of statistical errors on the estimation of straight lines in noisy lidar data [13].

\section{APPENDIX I}

\section{FORMULAS FOR THE ERRORS GIVEN IN TABLE I}

The formulas for Table I are derived from the scanning angle $\xi(t)$ and the line segment's motion given by $y(t)$

$$
\begin{aligned}
& \xi(t)=\xi_{t_{F}}+\dot{\xi} \cdot\left(t-t_{F}\right) \\
& y(t)=d_{t_{F}}+v_{\mathrm{rel}} \cdot\left(t-t_{F}\right)
\end{aligned}
$$

with $\dot{\xi}=\Delta \xi / \Delta t$. The time-continuous scanning of the line segment which can be seen in Fig. 4 starts at time $t_{S}$ when the scan ray hits the right corner of the car, $\left(w / 2, y\left(t_{S}\right)\right)$

$$
\xi\left(t_{S}\right)=-\arctan \left(\frac{w / 2}{y\left(t_{S}\right)}\right)
$$

and it ends at time $t_{E}$ when the scan ray hits the left corner, $\left(-w / 2, y\left(t_{E}\right)\right)$

$$
\xi\left(t_{E}\right)=\arctan \left(\frac{w / 2}{y\left(t_{E}\right)}\right)
$$

The LS estimates of distance $d$, heading angle $\psi$ and width $w$ are computed for the $N$ measurements $\left(x_{i}, y_{i}\right)$ taken between $t_{S}$ and $t_{E}\left[(\cdot)^{\dagger}\right.$ denotes the matrix pseudoinverse $]$

$$
\begin{aligned}
\left(\begin{array}{c}
\tilde{d} \\
\tan \tilde{\psi}
\end{array}\right) & =\left(\begin{array}{cc}
1 & x_{1} \\
\vdots & \vdots \\
1 & x_{N}
\end{array}\right)^{\dagger} \cdot\left(\begin{array}{c}
y_{1} \\
\vdots \\
y_{N}
\end{array}\right) \\
\tilde{w} & =\left\|\left(\begin{array}{c}
x_{N} \\
y_{N}
\end{array}\right)-\left(\begin{array}{c}
x_{1} \\
y_{1}
\end{array}\right)\right\|_{2} .
\end{aligned}
$$

The errors are given by the difference of the LS estimates and the nominal values, e.g., $\Delta \tilde{d}=\tilde{d}-d_{t_{F}}$. Equation (21) can be linearized for small relative velocities yielding simple formulas for the errors

$$
\begin{aligned}
& \Delta \tilde{d} \approx-v_{\text {rel }}\left(t_{F}-\frac{t_{S}+t_{E}}{2}\right) \\
& \Delta \tilde{\psi} \approx \arctan \left(\frac{v_{\mathrm{rel}}\left(t_{E}-t_{S}\right)}{w}\right) \\
& \Delta \tilde{w} \approx w\left(\frac{1}{\cos \Delta \tilde{\psi}}-1\right) .
\end{aligned}
$$

\section{APPENDIX II \\ FORMULAS FOR THE ERRORS GIVEN IN TABLE II}

The formulas are similar to those given in Appendix I except for the position of the car's corners. They are shifted by the lane width $W=3.2 \mathrm{~m}$ and located at $(-W+w / 2, y(t))$ and $(-W-w / 2, y(t))$. Therefore, times $t_{S}$ and $t_{E}$ are different

$$
\begin{aligned}
& \xi\left(t_{S}\right)=\arctan \left(\frac{W-w / 2}{y\left(t_{S}\right)}\right) \\
& \xi\left(t_{E}\right)=\arctan \left(\frac{w / 2+W}{y\left(t_{E}\right)}\right) .
\end{aligned}
$$

This results in different scan points $\left(x_{i}, y_{i}\right)$ and thus in different estimates for $d, \psi$ and $w$. The formulas for $\psi$ and $w$ remain the same [cf. (21)], but the formula for $d$ has to account for the offset

$$
\tilde{d}_{\text {correct }}=\tilde{d}-W \tan \tilde{\psi} \text {. }
$$

\section{APPENDIX III}

\section{LINE SEGMENT MODEL OF THE REAR AND FRONT}

Since the rear and front are orthogonal to the current velocity vector (cf. Fig. 2 and the assumptions made in Section II-A), the following holds:

$$
m=\tan \psi \text {. }
$$

Even though any point of the line has to fulfill (5) at any time $t \in\left[t_{S}, t_{E}\right]$, the center of the rear, respectively, front is a good choice when modeling the line segment

$$
y_{c}(t)=m x_{c}(t)+c(t), \quad t \in\left[t_{S}, t_{E}\right]
$$

Replacing $m$ by (24) and using (3) with the transformation (4) yields

$$
\begin{aligned}
c(t)= & y_{c . t_{F}}+\left(v \cos \psi-v_{s}\right)\left(t-t_{F}\right) \\
& -\tan \psi\left(x_{c . t_{F}}-v \sin \psi\left(t-t_{F}\right)\right) \\
= & -\tan \psi x_{c . t_{F}}+y_{c . t_{F}} \\
& +(\underbrace{(\cos \psi+\tan \psi \sin \psi)}_{=1 / \cos \psi}-v_{s})\left(t-t_{F}\right) .
\end{aligned}
$$

Putting (26) into (5) gives the time-variant line model

$$
y=\tan \psi\left(x-x_{c . t_{F}}\right)+y_{c . t_{F}}+\left(\frac{v}{\cos \psi}-v_{s}\right)\left(t-t_{F}\right) \text {. }
$$


Equation (8b) is applicable when both corners are visible. When only one corner is visible and the position of the center is known, width $w$ is determined by twice the distance between center and visible corner $\left(t_{*} \in\left\{t_{S}, t_{E}\right\}\right)$

$$
w=2\left\|\left(\begin{array}{l}
x\left(t_{*}\right) \\
y\left(t_{*}\right)
\end{array}\right)-\left(\begin{array}{c}
x_{c . t_{F}}-v \sin \psi\left(t_{*}-t_{F}\right) \\
y_{c . t_{F}}+\left(v \cos \psi-v_{s}\right)\left(t_{*}-t_{F}\right)
\end{array}\right)\right\|_{2} .
$$

\section{APPENDIX IV \\ Line SegMent Model OF THE Side}

A model similar to (5) is used for the line segment model of the side

$$
y_{d}(t)=m^{*} x_{d}(t)+c^{*}(t) \quad \text { with } m^{*}=\tan \psi^{*} .
$$

For the reasons given in Section III-A the motion is modeled by a linear model with constant velocity $v$ and angle $\psi^{*}$. This leads to

$$
\begin{aligned}
& x_{d}(t)=x_{d . t_{S}}+v \cos \psi^{*}\left(t-t_{S}\right) \\
& y_{d}(t)=y_{d . t_{S}}+\left(v \sin \psi^{*}-v_{s}\right)\left(t-t_{S}\right) .
\end{aligned}
$$

The transformation of the initial values can be carried out analog to (4).

Combining (28) and (29) gives

$$
\begin{aligned}
c^{*}(t)= & y_{d . t_{F}}+\left(v \sin \psi^{*}-v_{s}\right)\left(t-t_{F}\right) \\
& -\tan \psi^{*}\left(x_{d . t_{F}}+v \cos \psi^{*}\left(t-t_{F}\right)\right) \\
= & -\tan \psi^{*} x_{d . t_{F}}+y_{d . t_{F}}-v_{s}\left(t-t_{F}\right) .
\end{aligned}
$$

Putting (30) into (28) yields the line model

$$
y=\tan \psi^{*}\left(x-x_{d . t_{F}}\right)+y_{d . t_{F}}-v_{s}\left(t-t_{F}\right) .
$$

\section{REFERENCES}

[1] S. Ono, H. Kawasaki, K. Hirahara, M. Kagesawa, and K. Ikeuchi, "Egomotion estimation for efficient city modeling by using epipolar plane range image analysis," in Proc. 10th World Congr. Intelligent Transport Systems and Services (ITSWC 2003), 2003.

[2] S. Thrun, "Robotic mapping: A survey," in Exploring Artificial Intelligence in the New Millenium, G. Lakemeyer and B. Nebel, Eds. San Mateo, CA: Morgan Kaufmann, 2002, ch. 1.

[3] D. Hähnel, W. Burgard, and S. Thrun, "Learning compact 3D models of indoor and outdoor environments with a mobile robot," Robot. Autonom. Syst., vol. 44, no. 1, pp. 15-27, 2003.

[4] I. R. MacDonald, J. S. Chu, J. F. Reilly II, M. Blincow, and D. Olivier, "Deep-ocean use of the sm2000 laser line scanner on submarine nr-1 demonstrates system potential for industry and basic science," in Conf. Proc. OCEAN '05, MTS/IEEE Challenges of Our Changing Global Environment, 1995, vol. 1, pp. 555-565, IEEE.
[5] R. Behringer, S. Sudareswaran, B. Gregory, R. Elsley, B. Addison, W. Guthmiller, R. Daily, and D. Bevly, "The DARPA grand challenge-Development of an autonomous vehicle," in Proc. 2004 IEEE Intelligent Vehicles Symp., 2004, pp. 226-231, IEEE.

[6] A. E. Johnson and A. M. San Martin, "Motion estimation from laser ranging for autonomous comet landing," in Proc. 2000 IEEE Int. Conf. Robotics and Automation, ICRA '00, 2000, vol. 1, pp. 132-138.

[7] C. Rasmussen, "Combining laser range, color, and texture cues for autonomous road following," in Proc. 2002 IEEE Int. Conf. Robotics and Automation (ICRA '02), 2002, vol. 4, pp. 4320-4325.

[8] N. Kaempchen and K. Dietmayer, "Fusion of laserscanner and video for advanced driver assistance systems," in Proc. ITS 2004, 11th World Congr. Intelligent Transportation Systems, Nagoya, Japan, 2004, ITS.

[9] P. Ballard and F. Vacherand, "Simulation and understanding of range images acquired in fast motion," in Proc. 1994 IEEE Int. Conf. Robotics and Automation, 1994, vol. 3, pp. 2242-2247.

[10] F. Blais, M. Picard, and G. Godin, "Accurate 3D acquisition of freely moving objects," in Proc. 2nd Int. Symp. 3D Data Processing, Visualization and Transmission 2004, 2004, pp. 422-429, (3DPVT).

[11] G. Gräfe, Grün/Kahmen, Ed., "Mobile mapping with laser scanners using the MoSES," in Optical 3-D Measurement Techniques VI, Zürich, Switzerland, 2003, vol. I, pp. 381-388.

[12] J. L. Martínez, A. Mandow, J. Morales, A. García-Cerezo, and S. Pedraza, "Kinematic modelling of tracked vehicles by experimental identification," in Proc. 2004 IEEE/RSJ Int. Conf. Intelligent Robots and Systems, IROS '04, 2004, pp. 1487-1492.

[13] A. Kapp and L. Gröll, "Optimal estimation of line segments in noisy lidar data," in Signal Process., 2006, vol. 86, no. 9, pp. 2304-2317.

[14] J.-S. Gutmann, T. Weigel, and B. Nebel, "A fast, accurate, and robust method for self-localization in polygonal environments using laserrange-finders," Adv. Robot. J., vol. 14, no. 8, pp. 651-668, 2001.

[15] V. Nguyen, A. Martinelli, N. Tomatis, and R. Siegwart, "A comparison of line extraction algorithms using 2D laser rangefinder for indoor mobile robotics," in Proc. Int. Conf. Intelligent Robots and Systems 2005 (IROS 2005), 2005, pp. 1929-1934.

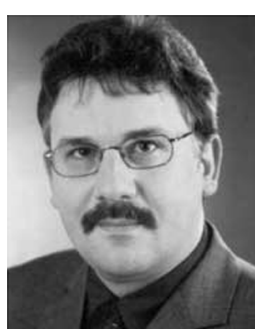

Lutz Gröll received the Dipl.-Ing. and Dr.-Ing. degrees from the Technische Universität Dresden, Dresden, Germany, in 1989 and 1995, both in electrical engineering.

Since 2001 he has been the Head of the research group "Process Modeling and Control Engineering" at the Institut für Angewandte Informatik, Forschungszentrum Karlsruhe, Karlsruhe, Germany. His research interests are in parameter identification, nonlinear control, and optimization theory.

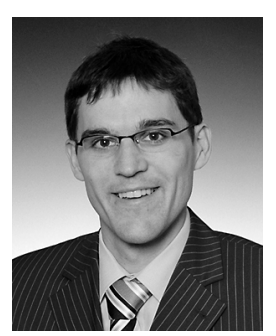

Andreas Kapp (S'05) received the M.Sc. degree in communications, control, and digital signal processing with distinction from the University of Strathclyde, Glasgow, U.K. in 2002. He is currently pursuing the Dr.-Ing. degree at the Institut für Mess-und Regelungstechnik, Universität Karlsruhe, Karlsruhe, Germany.

His research interests are in signal processing for lidar scanners and parameter identification. 